\title{
Resonance crossing experiment at a proof of principle fixed field alternating gradient accelerator
}

\author{
Masamitsu Aiba* \\ Department of Physics, Graduate School of Science, University of Tokyo, 7-3-1 Hongo, Bunkyo-ku, Tokyo 113-0033, Japan \\ Shinji Machida ${ }^{\dagger}$ and Yoshiharu Mori ${ }^{\ddagger}$ \\ High Energy Accelerator Research Organization (KEK), 1-1 Oho, Tsukuba-shi, Ibaraki 305-0801, Japan \\ Shoroku Ohnuma \\ Department of Physics and Astronomy, University of Hawaii at Manoa, Honolulu, Hawaii 96822, USA
}

(Received 28 March 2006; published 14 August 2006)

\begin{abstract}
An experimental study of crossing a third order uncoupled resonance was performed in a proof of principle fixed field alternating gradient with various widths of resonance and crossing speed. We observed that part of the beam is transported to a larger amplitude when the crossing speed is relatively small. We derived an "adiabatic parameter" from a "particle trapping" model as a useful index for avoiding beam deterioration due to resonance crossing. It relates the crossing speed to the resonance width and the amount of nonlinear detuning. When the adiabatic parameter is more than 7 , resonance crossing will not affect a beam.
\end{abstract}

DOI: 10.1103/PhysRevSTAB.9.084001

PACS numbers: 29.20. $-\mathrm{c}, 29.27 .-\mathrm{a}, 41.75 .-\mathrm{i}$

\section{INTRODUCTION}

Fixed field alternating gradient (FFAG) accelerators have in principle constant betatron tunes when the geometrical and optical scalings are realized with a scaled radial dependence of magnetic field [1,2]. However, tunes may change slightly because of fringing field and flux saturation in iron poles. Even when strong resonances such as integer, half integer, and intrinsic resonances are avoided, tunes may cross higher order resonances. At the same time, if the condition of constant tune is abandoned, different designs of FFAG become possible. Such machines are called nonscaling FFAG [3] in contrast to the original scaling FFAG. Tunes then cross resonances, even strong resonances, repeatedly. For both types of scaling and nonscaling FFAG, resonance crossing has become an important issue in beam dynamics as we must avoid unacceptable deterioration in beam quality.

For scaling type of FFAG, the nonlinear detuning, i.e., the dependence of tunes on the oscillation amplitudes, is significant and its effect cannot be ignored. Since nonscaling FFAG is basically composed of linear elements, nonlinear detuning may not be as significant. However, the fringing field and allowed poles will introduce nonlinear components. Then nonlinear detuning may not be totally negligible for resonance crossing.

\footnotetext{
*Present address: High Energy Accelerator Research Organization, 1-1 Oho, Tsukuba-shi, Ibaraki 305-0801, Japan.

Present address: Rutherford Appleton Laboratory, Chilton, Didcot, Oxfordshire, OX11 0QX, UK.

${ }^{\ddagger}$ Present address: Kyoto University Research Reactor Institute, 2-1010 Asashironishi, Kumatori-cho, Sennan-gun, Osaka 5900494, Japan.
}

In this paper, we study the resonance crossing with dominant nonlinear detuning. In the past, there have been several studies concerning resonance crossing [4-8]. Among them, Chao and Month have proposed a model of "particle trapping" [8] in which some particles of a beam are trapped and subsequently transported to larger amplitudes when a resonance is crossed in the presence of dominant nonlinear detuning. There is one experimental study that has been carried out at CERN [9] for a beam extraction based on particle trapping. This work, however, does not answer all the questions that we should know, for instance, how large crossing speed should be and how much driving term would be permitted. We have performed a resonance crossing experiment, a single passage across $3 \nu_{x}=7$, with PoP (proof of principle) FFAG to clarify these points. ${ }^{1}$

\section{EXPERIMENT WITH POP FFAG}

\section{A. PoP FFAG and its remodeling}

A resonance crossing of the one-dimensional uncoupled third order resonance, $3 \nu_{x}=7$, has been studied with PoP FFAG. Figure 1 is a picture of PoP FFAG and its main parameters are summarized in Table I. PoP FFAG constructed at KEK [11] is the world's first proton FFAG accelerator. Through commissioning and subsequent machine studies [12], we have come to understand its basic properties. Since peak beam current of PoP FFAG is a few $\mathrm{nA}$, the space charge detuning is totally negligible even

\footnotetext{
${ }^{1}$ This study is part of the dissertation work by one of the authors [10]. In this paper, we discuss only the crossing in one direction. Crossing in the other direction is discussed in Refs. [4,10].
} 


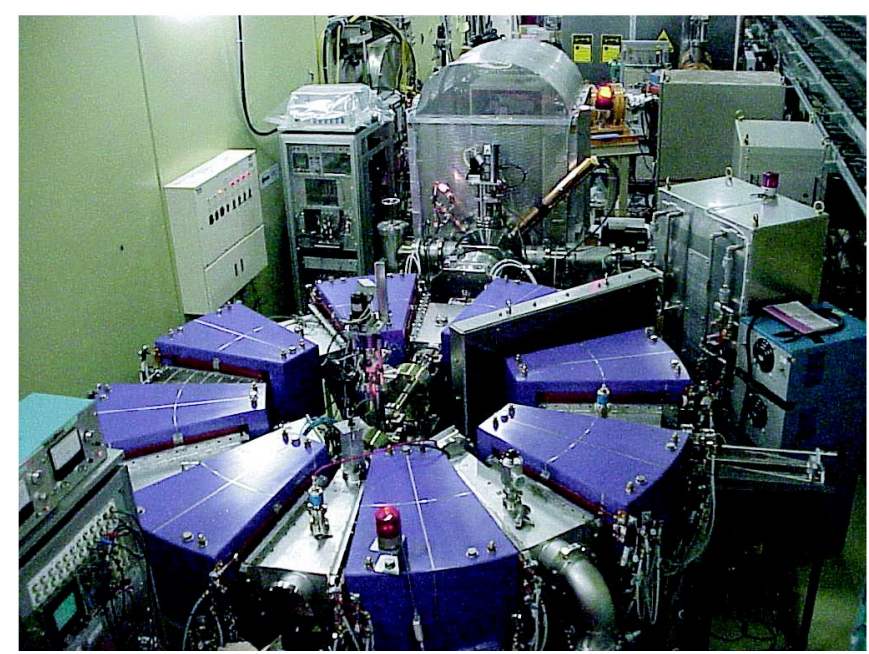

FIG. 1. (Color) A picture of PoP FFAG.

though the injection energy is very low. Beam dynamics in this experiment can be treated as single particle dynamics.

PoP FFAG is designed as a scaling FFAG of which magnetic field on the median plane is expressed by

$$
B=B_{0}(\theta)\left(\frac{r}{r_{0}}\right)^{k}
$$

where $B_{0}(\theta)$ is the field strength at the machine radius $r=$ $r_{0}, \theta$ is the azimuthal angle, and $k$ is the field index or the so-called $k$-value. Signs of $B_{0}(\theta)$ are opposite in focusing and defocusing magnets to realize alternating gradient. It is obvious that such a field has nonlinear components. With this field, a zero chromaticity condition is expected, and tunes are almost constant from injection to the final energy as shown in Table I.

Magnets of PoP FFAG were modified for this study so as to let the horizontal tune cross the resonance, $3 \nu_{x}=7$. The method used for this purpose is to insert iron plates between the upper and lower parts of magnet as shown in Fig. 2. With iron plates of $4 \mathrm{~mm}$ thickness, the gap of magnet becomes widened and the field is distorted from the one given in Eq. (1). Iron plates were installed in all eight magnets so that super periodicity is maintained.

TABLE I. Parameters of PoP FFAG. The ranges of values are the variation from injection to the final energy.

\begin{tabular}{lc}
\hline \hline Parameter & Value \\
\hline Number of cells & 8 \\
Lattice structure & DFD triplet \\
$k$-value & 2.5 \\
Magnetic field & $0.14-0.32 \mathrm{~T}(\mathrm{~F}), 0.04-0.13 \mathrm{~T}(\mathrm{D})$ \\
Beam energy & $50-500 \mathrm{keV}$ \\
Average radius & $0.81-1.14 \mathrm{~m}$ \\
Betatron tune & $0.61-1.40 \mathrm{MHz}$ \\
Revolution frequency & $2 \mathrm{kV}$ \\
rf voltage & $1 \mathrm{~ms}$ \\
Acceleration period & \\
\hline \hline
\end{tabular}

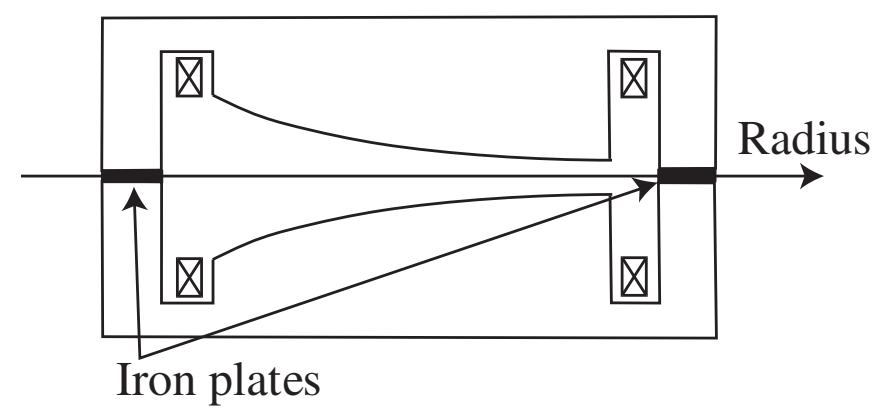

FIG. 2. Schematic view of inserted ion plates. The thickness of the iron plate is $4 \mathrm{~mm}$.

The modification using iron plates works as intended. Figure 3(A) shows betatron tunes in tune space before and after the modification, and the beam energy dependence of horizontal tune, calculated as well as measured, is given in Fig. 3(B). The horizontal tune crosses the resonance of $3 \nu_{x}=7$ around $130 \mathrm{keV}$ during acceleration. The machine radius at the straight section corresponding to this energy is about $0.89 \mathrm{~m}$.

\section{B. Crossing with rf acceleration}

Since rf voltage is very high relative to the beam energy, it is possible to examine a wide range of energy gain per turn, resulting in a wide range of crossing speed. Magnetic alloy (MA) cores having a broad band impedance and a high permeability [13] are used for rf cavity to accelerate protons. One notable feature of the machine is a fast acceleration in less than $1 \mathrm{~ms}$. From the known slope of tune to the beam energy, the resonance crossing speed is determined as the product of the slope and energy gain per turn. The energy gain per turn was varied from 0.13 to $1.56 \mathrm{keV} /$ turn in this study.

Because of synchrotron oscillation, it is not possible to have the same crossing speed for all particles in a bunched beam. For an unambiguous observation, the fluctuation of crossing speed was controlled to be as small as possible. Two methods were employed to reduce the fluctuation of crossing speed.

(1) Make bunch length as short as possible.

(2) Inject the beam at the center of rf bucket.

The beam chopper was tuned to chop out a short bunch of less than $100 \mathrm{~ns}$. This length corresponds to the rf phase of 20 degrees at injection energy. In order to inject a beam into the center of rf bucket, a mountain view plot was used for beam tuning. Figure 4 shows examples of mountain view plot. During data acquisition, the mountain view plot was regularly checked to confirm that the dipole oscillation is acceptably small.

\section{Driving term of resonance}

A variable source of closed orbit distortion (COD) was introduced to control the width of resonance. The resonance of $3 \nu_{x}=7$ is not intrinsic with the super periodicity 

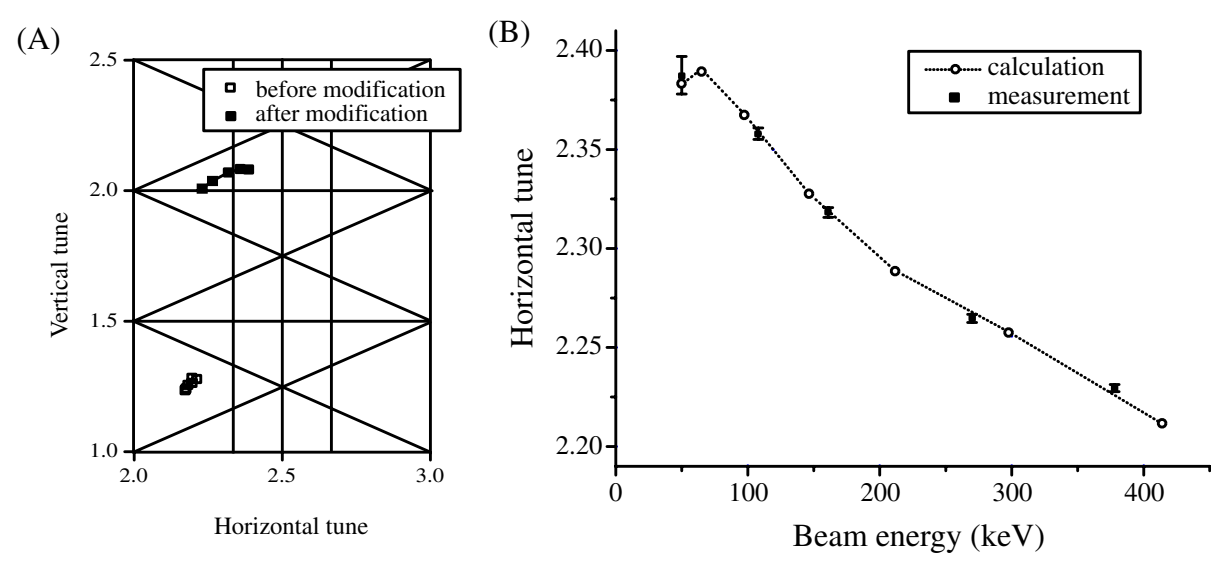

FIG. 3. Measured betatron tunes. (A) Betatron tunes in tune space. (B) Horizontal tune vs beam energy (after modification). In (A), FD ratio before the modification is the nominal value of 3.9 and FD ratio after the modification is 2.43 . The FD ratio is simply a ratio of focusing field to defocusing field. The errors of measured tunes are due to resolution of fast Fourier transform.
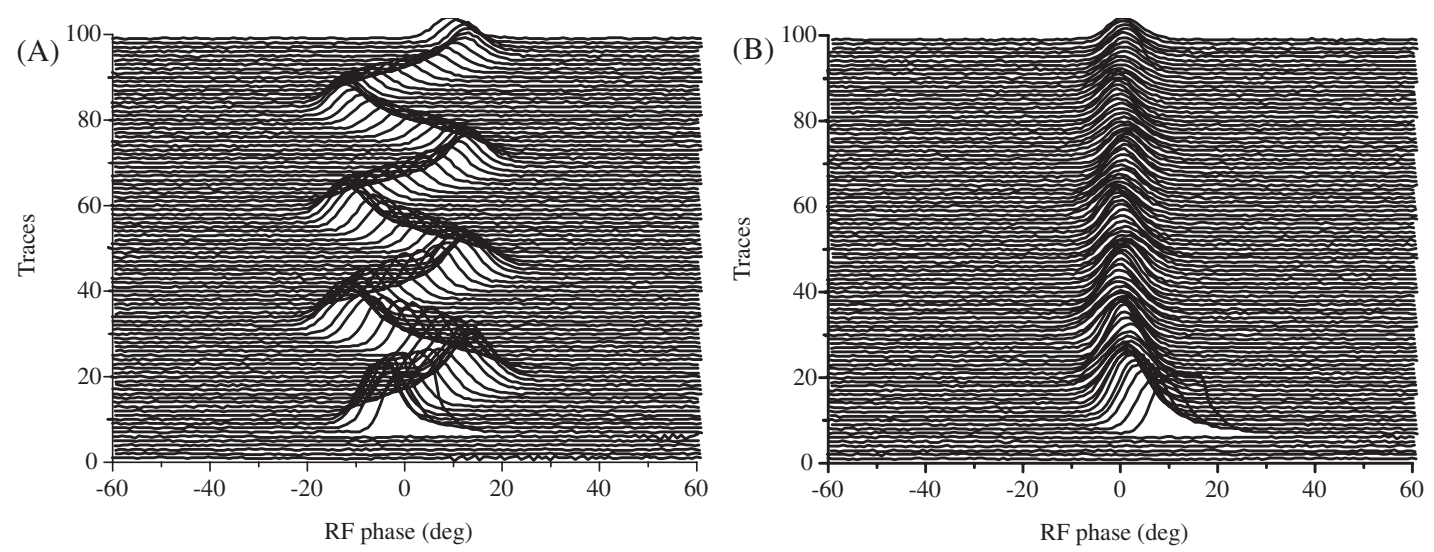

FIG. 4. Mountain view plots. (A) Off-timing injection. (B) On-timing injection. These plots are from injection tuning; injection timing is out of tuning in (A), and after tuning in (B). The difference of injection timing between (A) and (B) is about $60 \mathrm{~ns}$.

of eight. As long as the super periodicity is retained, this resonance will not be excited. In order to break the super periodicity, two cells separated by three cells were selected to be the source of COD. Coil currents of focusing magnets of these two cells were set at different values from the others. For the sake of convenience, we will call this difference in coil currents "current error" throughout this paper. The reason for using a pair of magnets as the error source, instead of one, is to keep the angle of COD unchanged at the injection septum. For beam injection, the angle between COD and the injection septum should be at its optimum value. The locations of two magnets are third and sixth from injection septum. With this symmetrical arrangement, the angle can be maintained at any magnitude of COD. The current errors of 0\% (no current error), $-2 \%$, and $-3 \%$ were examined.

Another source of COD, which was not intended, is the cores of rf cavity installed at the center of straight section. They are penetrated by the fringe field of nearby magnets. The effect of this error source will be discussed in Sec. IVA 2.

\section{Beam measurement}

Two diagnostic devices were used in the study, a beam position monitor (BPM) and a beam scraper. Figure 5 is a schematic drawing of BPM electrodes. The BPM is avail-

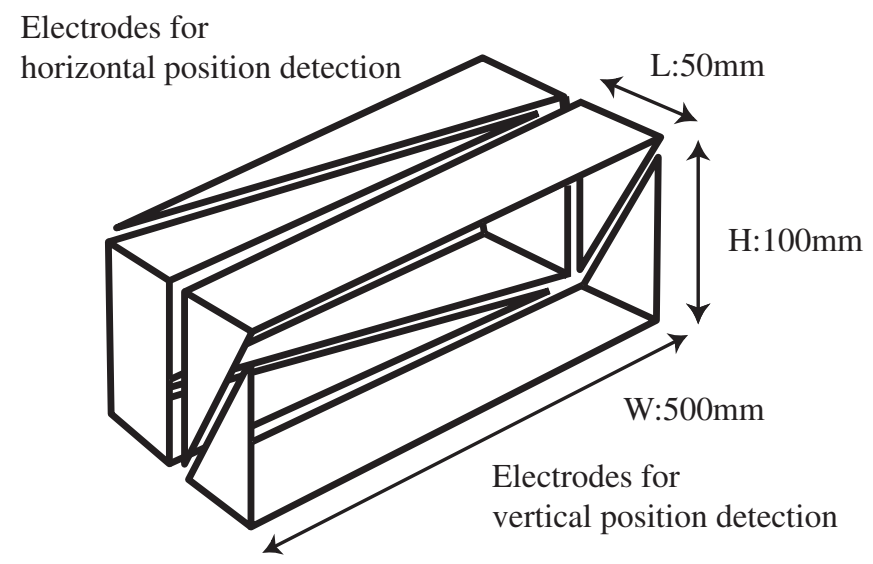

FIG. 5. Geometry of beam position monitor electrodes. 

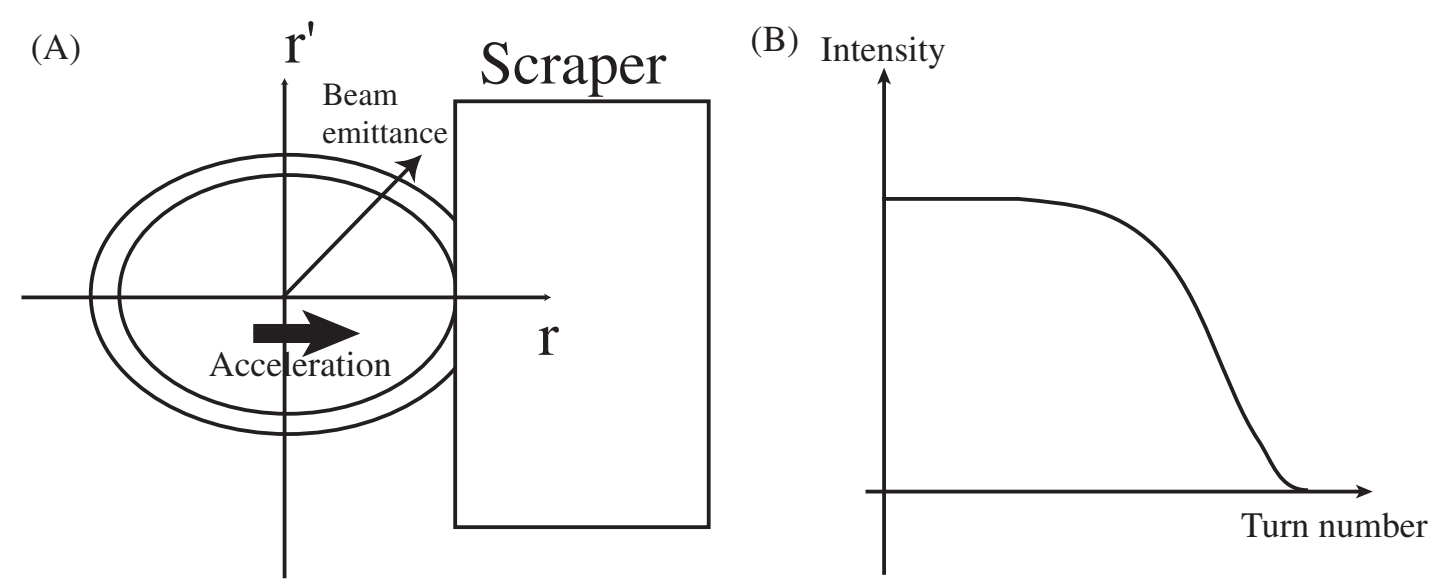

FIG. 6. Method of beam measurement. (A) Beam scraping. (B) Beam intensity during scraping.

able not only to detect the position of beam centroid but to measure the tunes, bunch signal, and beam intensity as well. From the Fourier transform of signals from one electrode, tune is obtained. The summation of signals from either pair of electrodes corresponds to bunch signal and the beam intensity can be found from the time integral of bunch signals. The scraper was inserted horizontally from the outer side of the ring and its radial position can be changed to cover the orbit from injection to the final energy.

Figure 6 depicts the basic idea of the measurement. During acceleration, the orbit shifts toward the outer radius and the beam is scraped gradually by the scraper placed at an observation position. A decreasing curve of the beam intensity contains information of the particle distribution in beam emittance. The scraper position was changed to measure beams before and after resonance crossing.

\section{DATA ANALYSIS AND RESULTS}

\section{A. Correcting beam loss due to residual gas}

Since the beam energy of PoP FFAG is low, beam loss due to the residual gas is not negligible, especially near injection energy. A correction of beam loss is necessary as the beam loss continues during beam scraping. The dominant process of beam loss is a charge transfer in which a charged particle loses its charge and is lost through a single process. Note that this process decreases the beam intensity without affecting the beam emittance. Beam intensity curves were corrected taking into account the effect of charge transfer. Details of the correction are given in the Appendix. Figure 7 shows an example of observed intensity and the corresponding intensity normalized by the correction.

\section{B. Beam emittance}

Beam emittance must be taken into account in this study because we assume dominant nonlinear detuning. A difference in oscillation amplitude will result in different single particle motion. In order to obtain beam emittance, a normalized intensity as a function of the beam emittance is needed. For this process, the length of orbit shift during acceleration and the beta function are necessary. The relation between the beam energy and beam position is known through previous machine studies [12]. As shown in Fig. 8,
(A)

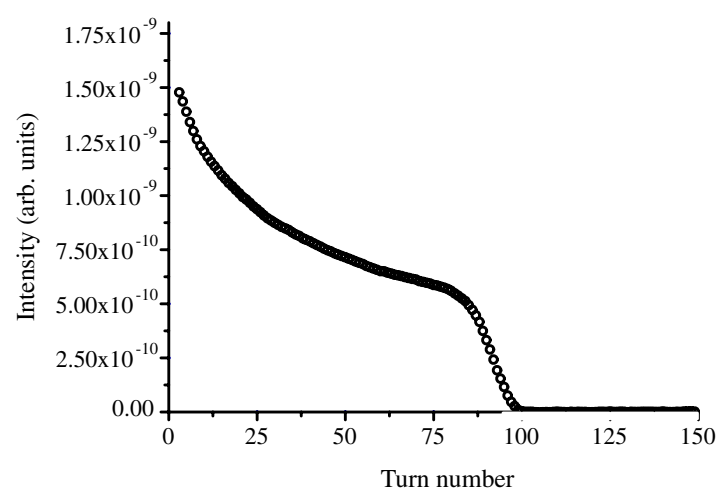

(B)

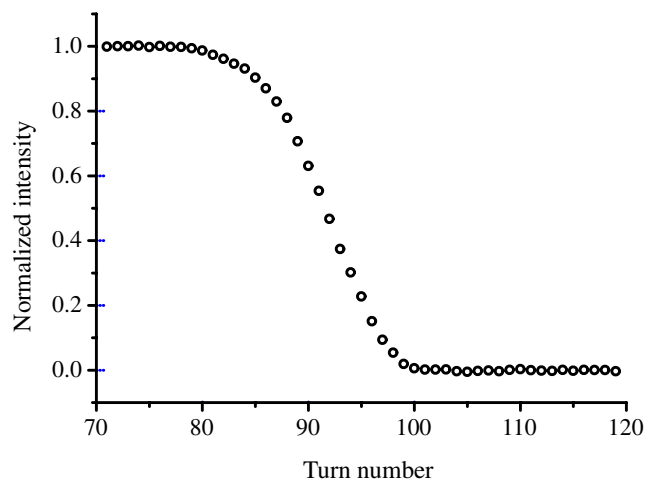

FIG. 7. Correction for beam loss due to residual gas. (A) Raw intensity data. (B) Normalized intensity after correction. For the current error of $-2 \%$, the energy gain of $1.56 \mathrm{keV} /$ turn, the scraper position of $948 \mathrm{~mm}$. 


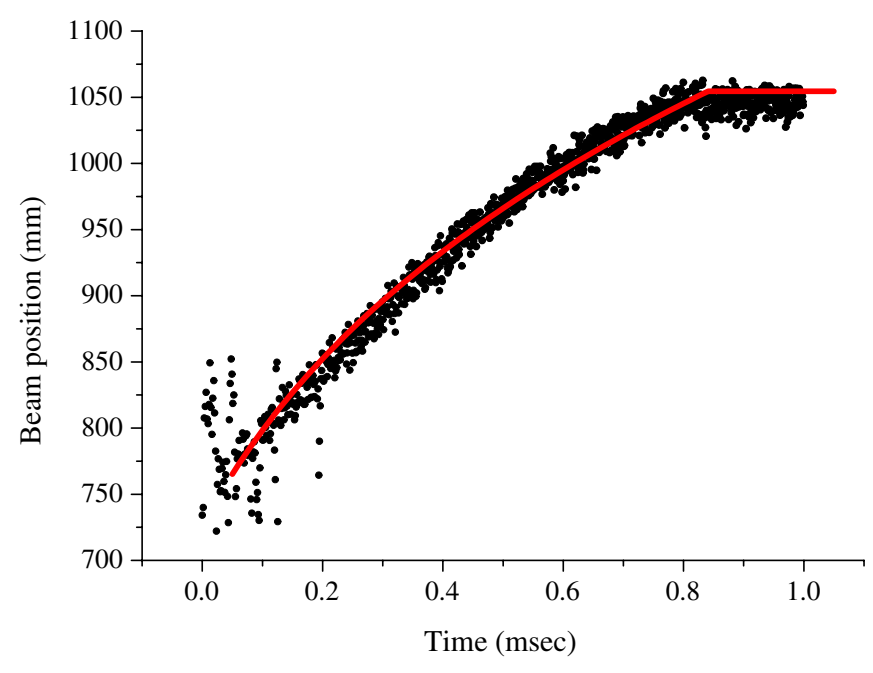

FIG. 8. (Color) Orbit shift during acceleration. (Data is taken from Ref. [12].)

the measured beam position during acceleration shows good agreement with calculation. Since the beta function cannot be obtained experimentally at PoP FFAG, calculated values with simulation described in Sec. IVA 2 are employed. Figure 9 shows the normalized intensity versus the beam emittance at $50 \mathrm{keV}$ (injection) and $110 \mathrm{keV}$ when the current error is $-2 \%$. Since the injection lasts only about four turns, the particle distribution is not smooth at injection. It is, however, smooth at $110 \mathrm{keV}$ after many turns. The beam emittance at the $87 \%$ level, for example, will therefore change during acceleration not from the adiabatic damping but from the smoothing process. The beam emittances at $110 \mathrm{keV}$ will be used for the discussion in Sec. IV as it is reasonable to use the intensity data after the smoothing process and just before crossing resonance. The $87 \%$ beam emittances at $110 \mathrm{keV}$ were

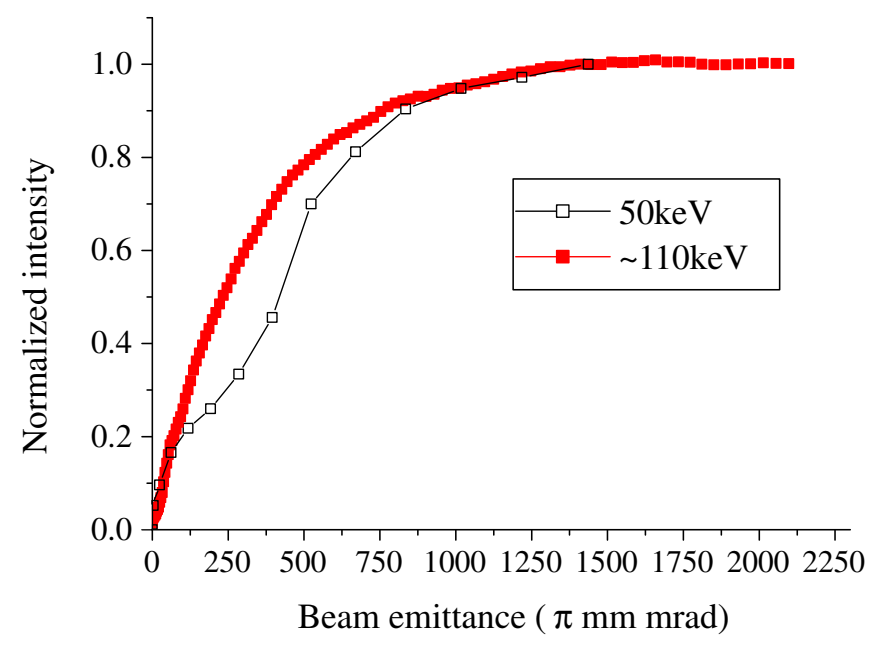

FIG. 9. (Color) Beam emittance for injection energy and just before crossing for the current error of $-2 \%$. In the beam tuning, $87 \%$ beam emittance was expected to be about $800 \pi \mathrm{mm} \mathrm{mrad}$ at injection energy with multiturn injection.

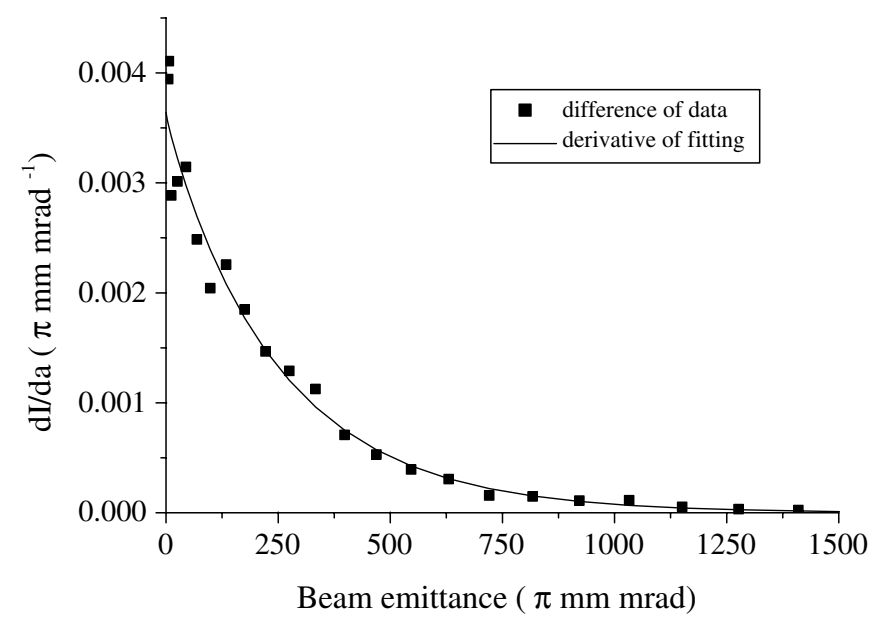

FIG. 10. Particle distribution in beam emittance. For the current error of $-2 \%$, the energy gain of $1.56 \mathrm{keV} /$ turn, the scraper position of $948 \mathrm{~mm}$. The vertical axis is the derivative or difference of the normalized intensity $I$ with respect to $a$, where $a$ is the beam emittance divided by $\pi$.

obtained assuming Gaussian distribution. They were $830 \pi \mathrm{mm}$ mrad, $660 \pi \mathrm{mm} \mathrm{mrad}$, and $790 \pi \mathrm{mm} \mathrm{mrad}$, respectively, for the current error of $0 \%,-2 \%$, and $-3 \%$.

\section{Particle distribution in beam emittance}

A particle distribution in beam emittance can be obtained by differentiating normalized intensity as a function of beam emittance. Figure 10 shows the particle distribution obtained from the normalized intensity curve of Fig. 7. With particle distributions in beam emittance before and after crossing, an effect of resonance crossing will become clear.

\section{Results for fast and slow crossings}

Figure 11 shows two typical results, for fast $(A, B)$ and slow $(C, D)$ crossings. The curves of normalized intensity are quite similar in shape when the crossing speed is large. The particle distribution in beam emittance is concentrated at the beam center in the same way. From this, one can conclude that there is no substantial effect on the beam arising from the resonance crossing when the crossing speed is sufficiently large. In contrast, a two-tier structure is clearly observed in the normalized intensity curve when the crossing is slow. As shown in Fig. 11(D), part of the beam is transported to a larger amplitude after crossing. Transported particles will eventually be lost. From the twotier structure, the ratio of the number of transported particles to the total number of particles can be obtained. Table II summarizes the ratios obtained in this study.

\section{DISCUSSION}

\section{A. Comparison of trapping efficiencies}

We clearly observed that there is no substantial effect on the beam with relatively fast crossing but part of the beam 

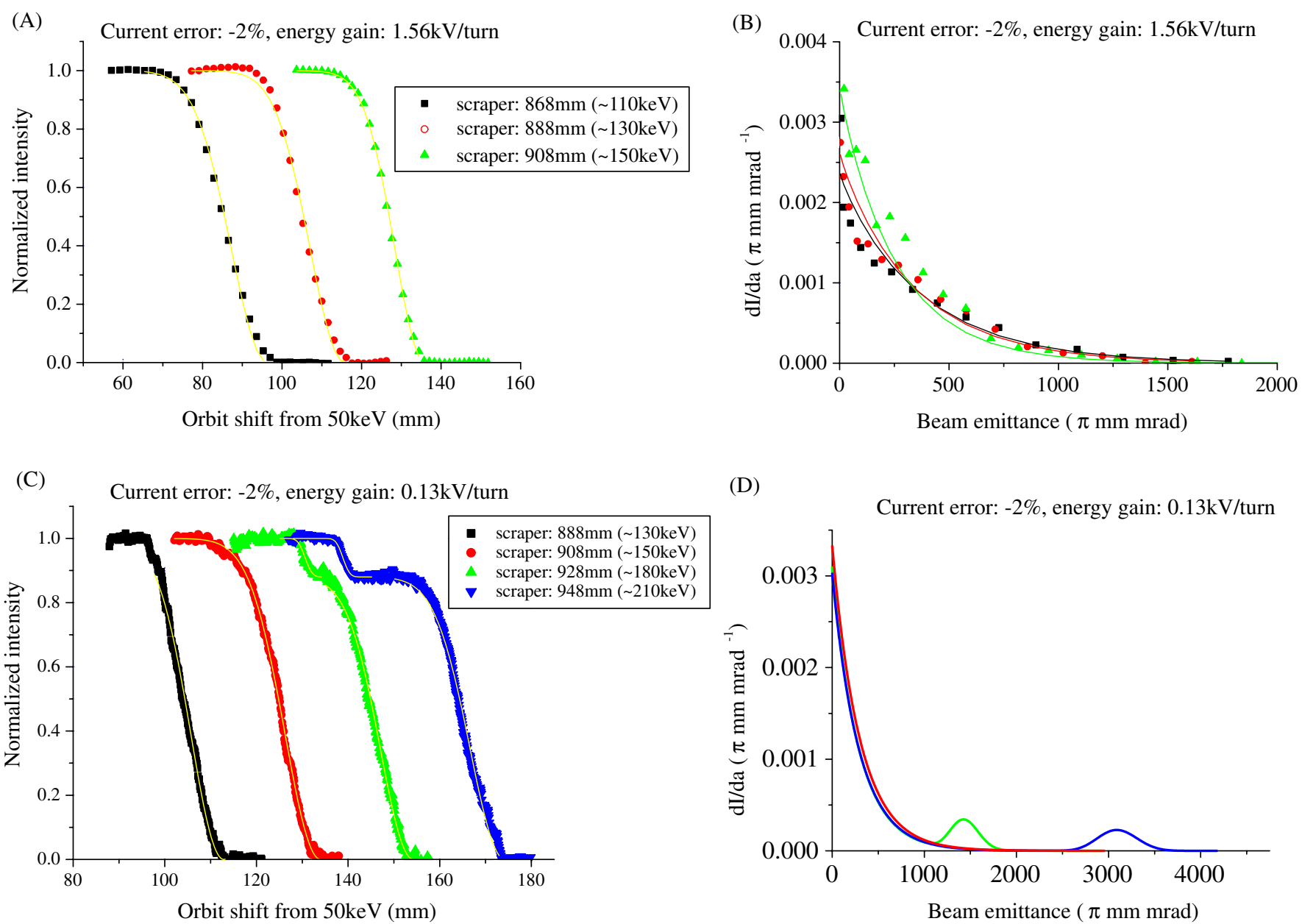

FIG. 11. (Color) Typical results for fast and slow crossings. (A) Normalized intensity (fast). (B) Particle distribution (fast). (C) Normalized intensity (slow). (D) Particle distribution (slow). The horizontal axis of the normalized intensity is transferred from the number of turns to the length of orbit shift from $50 \mathrm{keV}$ (injection).

is transported to a larger amplitude with relatively slow crossing. We will discuss the obtained ratios of transported particles through quantitative comparison between experimental data, theoretical model, and the prediction from simulation. The theoretical model and the method of simulation are explained below.

\section{1. "Particle trapping"}

We applied a model of "particle trapping" [8] in which "islands" in phase space may trap some particles and

TABLE II. Transported particle ratios in experiment. For energy gain more than $0.49 \mathrm{keV} /$ turn, a two-tier structure was not observed. The errors are evaluated from the ambiguity in the flat part of the two-tier structure.

\begin{tabular}{lcc}
\hline \hline Energy gain & $0.13 \mathrm{keV} /$ turn & $0.21 \mathrm{keV} /$ turn \\
\hline Current error $0 \%$ & $17.0 \pm 1.3$ & $11.9 \pm 1.5$ \\
$-2 \%$ & $12.0 \pm 3.7$ & $8.0 \pm 1.5$ \\
$-3 \%$ & $22.3 \pm 2.4$ & $15.3 \pm 3.4$ \\
\hline \hline
\end{tabular}

transport them to larger amplitudes when a high order resonance is crossed in the presence of dominant nonlinear detuning. An island is a stable region in phase space outside the central stable area and the number of such islands is the same as the order of the resonance. This model of particle trapping should be applicable to our case.

In order to apply their model, which is specifically for fifth order resonance, to our case, analytical expressions for the third order resonance have been derived following their treatment. First, we consider the phase space topology of uncoupled third order resonance in the presence of nonlinear detuning due to octupole. The single particle motion of betatron oscillation can be written as

$$
y(\theta)=[a(\theta) \beta(\theta)]^{1 / 2} \cos [\nu \theta+\varphi(\theta)],
$$

where $y$ is the displacement from closed orbit, $\theta$ is the independent variable of azimuthal angle, $\nu$ is the betatron tune, and $\beta$ is the beta function. Canonical variables $a$ and $\varphi$ satisfy the equations of motion 


$$
\begin{gathered}
a^{\prime}=-\frac{2}{\nu \beta} \frac{\partial H^{(1)}}{\partial \varphi}, \\
\varphi^{\prime}=\frac{2}{\nu \beta} \frac{\partial H^{(1)}}{\partial a},
\end{gathered}
$$

where $a^{\prime}$ and $\varphi^{\prime}$ are the derivatives with respect to $\theta$, and $H^{(1)}$ is the perturbation term of Hamiltonian. Obviously, $a$ and $\varphi$ are constants of motion in the absence of the perturbation term. For the uncoupled third order resonance, $H^{(1)}$ is

$$
H^{(1)}=O(\theta) y^{4}+S(\theta) y^{3},
$$

where $O(\theta)$ and $S(\theta)$ are proportional to the strength of octupole and sextupole, respectively. By using Eq. (2) for $y$ in Eq. (5), we find the phase-independent term and the term slowly varying in time:

$$
H^{(1)}=\nu \beta\left(3 B_{0} a^{2}+\left|A_{p}\right| a^{3 / 2} \cos 3 \psi\right)
$$

where

$$
\begin{gathered}
\left|A_{p}\right|=\frac{\left\langle\beta^{1 / 2}\right\rangle}{8 \pi \nu} \int_{0}^{2 \pi} d \theta e^{-i p \theta} S(\theta), \\
B_{0}=\frac{\langle\beta\rangle}{16 \pi \nu} \int_{0}^{2 \pi} d \theta O(\theta), \\
\psi=\left(\frac{1}{3} p-\nu\right) \theta-\varphi+\frac{1}{3} \eta,
\end{gathered}
$$

and $\eta$ is the phase factor, $\langle\beta\rangle$ is the average value of beta function. For the sake of simplicity, "smooth approximation" in which the beta function is assumed constant is employed here. The tune is close to the third order resonance excited by the $p$ th harmonic sextupole component. With Eqs. (3), (4), and (6),

$$
\begin{gathered}
a^{\prime}=-6\left|A_{p}\right| a^{3 / 2} \sin 3 \psi, \\
\varphi^{\prime}=12 B_{0} a+3\left|A_{p}\right| a^{1 / 2} \cos 3 \psi .
\end{gathered}
$$

For further convenience, we define

$a_{0}$ : the average emittance of the initial particle distribution divided by $\pi$,

$\alpha=a / a_{0}$ : the relative emittance,

$\Delta_{L}=\frac{1}{3} p-\nu:$ the linear tune shift,
$\Delta_{N L}=-12 B_{0} a_{0}$ : the nonlinear tune shift at $a_{0}$,

$\Delta_{e}=-3\left|A_{p}\right| a_{0}^{1 / 2}:$ the excitation width at $a_{0}$,

$B_{0}$ : the nonlinear detuning source (simply "nonlinear detuning"),

$\left|A_{p}\right|$ : the resonance driving term (simply "driving term"),

$\xi=3 \Delta_{L} / 2 \Delta_{e}$,

$\kappa=3 \Delta_{N L} / 4 \Delta_{e}$.

With these parameters, the above equations for $a$ and $\varphi$ become

$$
\begin{gathered}
\left(\alpha^{1 / 2}\right)^{\prime}=\Delta_{e} \alpha \sin 3 \psi, \\
\psi^{\prime}=\Delta_{L}+\Delta_{N L} \alpha+\alpha^{1 / 2} \Delta_{e} \cos 3 \psi
\end{gathered}
$$

An invariant of the motion is

$$
C=\xi \alpha+\kappa \alpha^{2}+\alpha^{3 / 2} \cos 3 \psi
$$

A particular value of $C$ defines a trajectory in phase space. The fixed points can be obtained by solving the following equations:

$$
\begin{aligned}
& \left.\frac{\partial C}{\partial \alpha}\right|_{f}=0, \\
& \left.\frac{\partial C}{\partial \psi}\right|_{f}=0,
\end{aligned}
$$

where $f$ is the coordinate $(\alpha, \psi)$ for fixed points. Whether these points are stable or unstable is determined as follows:

$$
\begin{gathered}
\left.\left.\frac{\partial^{2} C}{\partial \alpha^{2}}\right|_{f} \cdot \frac{\partial^{2} C}{\partial \psi^{2}}\right|_{f}>0 \text { : stable, } \\
\left.\left.\frac{\partial^{2} C}{\partial \alpha^{2}}\right|_{f} \cdot \frac{\partial^{2} C}{\partial \psi^{2}}\right|_{f}<0 \text { : unstable. }
\end{gathered}
$$

The fixed points are summarized in Table III, and Fig. 12 shows a phase space topology for various values of $\xi$.

During crossing the third order resonance, the phase space topology changes as shown in Fig. 12. It is seen that three islands are created and become larger as $\xi$ decreases. As shown in the last line of Table III, stable and unstable fixed points are almost at the same amplitude when $\kappa \gg 1$ and $\xi$ is not close to zero. Since the islands

TABLE III. Fixed points for third integer resonance $(\kappa>0)$.

\begin{tabular}{lcc}
\hline \hline Range of $\xi$ & Stable fixed points & Unstable fixed points \\
\hline$\xi>\frac{9}{32 \kappa}$ & $\alpha_{s}^{1 / 2}=0$ & None \\
$\xi=\frac{9}{32 \kappa}$ & $\alpha_{s}^{1 / 2}=0, \frac{3}{8 \kappa}$ & None \\
$\frac{9}{32 \kappa}>\xi>0$ & $\alpha_{s}^{1 / 2}=0, \frac{3}{8 \kappa}\left(1+\sqrt{1-\frac{32 \kappa}{9}}\right)$ & $\alpha_{u}^{1 / 2}=\frac{3}{8 \kappa}\left(1-\sqrt{1-\frac{32 \kappa}{9} \xi}\right)$ \\
$\xi=0$ & $\alpha_{s}^{1 / 2}=\frac{3}{4 \kappa}$ & $\alpha_{u}^{1 / 2}=0$ \\
$\xi<0$ & $\alpha_{s}^{1 / 2}=0, \frac{3}{8 \kappa}\left(1+\sqrt{1-\frac{32 \kappa}{9} \xi}\right)$ & $\alpha_{u}^{1 / 2}=\frac{3}{8 \kappa}\left(-1+\sqrt{1-\frac{32 \kappa}{9} \xi}\right)$ \\
\hline \hline
\end{tabular}



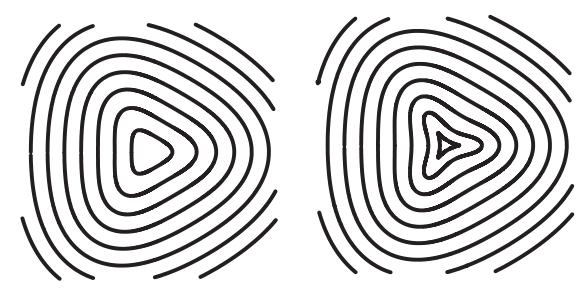

(b) $\xi=0.00281$

(a) $\xi=0.01$
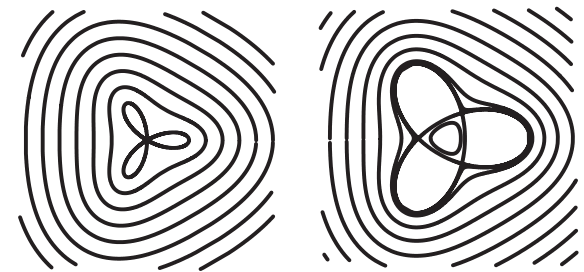

(d) $\xi=0$

(e) $\xi=-0.02$

FIG. 12. Phase space topology for third integer resonance, $\kappa=$ 100 .

are too small to trap the particle when $\xi$ is nearly zero, particles are mostly trapped at the amplitude for which the above approximation is valid. Then, $\alpha_{s}^{1 / 2}$ and $\alpha_{u}^{1 / 2}$ are approximately

$$
\alpha_{s}^{1 / 2} \approx \alpha_{u}^{1 / 2} \approx\left|\frac{\xi}{2 \kappa}\right|^{1 / 2}
$$

Equation (19) indicates that the shape of each island will be approximately an ellipse. The equation for the separatrix, which is the border of islands, is

$$
C=\xi \alpha_{u}+\kappa \alpha_{u}^{2}+\alpha_{u}^{3 / 2}=\xi \alpha+\kappa \alpha^{2}+\alpha^{3 / 2} \cos 3 \psi
$$

By taking $\cos 3 \psi=-1$, we can find $\alpha$ corresponding to the outer and inner edges of islands in the radial direction. We define them as $\alpha_{+}=\alpha_{u}+\delta_{+}, \alpha_{-}=\alpha_{u}+\delta_{-}$, respectively. Expand Eq. (20) keeping up to the second order of $\delta$. Then we find

$$
\delta_{ \pm} \approx \pm\left(\frac{2}{\kappa} \alpha_{u}^{3 / 2}\right)^{1 / 2},
$$

where $\left|\delta_{+}\right| \approx\left|\delta_{-}\right|$is assumed. The difference between two solutions $\delta_{+}-\delta_{-}$is the width of the island along the radial direction multiplied by $2 \alpha_{u}^{1 / 2}$;

$$
\delta_{+}-\delta_{-} \approx 2 \alpha_{u}^{1 / 2}\left(\alpha_{+}^{1 / 2}-\alpha_{-}^{1 / 2}\right) .
$$

The island width of phase direction is simply $\frac{2}{3} \pi \alpha_{u}^{1 / 2}$. Hence the total area of three islands is approximately

$$
A \approx 3 \pi\left(\frac{\alpha_{+}^{1 / 2}-\alpha_{-}^{1 / 2}}{2}\right)\left(\frac{2 \pi \alpha_{u}^{1 / 2} / 3}{2}\right) .
$$

With Eqs. (21)-(23), we find

$$
A \approx \frac{\pi^{2}}{\sqrt{2}} \kappa^{-(1 / 2)} \alpha_{s}^{3 / 4}
$$

When $\xi$ changes dynamically, the stable fixed points move outward at the rate

$$
\left(\alpha_{s}^{1 / 2}\right)^{\prime}=\frac{\xi^{\prime}}{3 / 2-4 \kappa \alpha_{s}^{1 / 2}} .
$$

From Eqs. (12) and (25), a criterion for adiabaticity is

$$
\left|\Delta_{e}\right| \alpha \gg \frac{\xi^{\prime}}{3 / 2-4 \kappa \alpha^{1 / 2}} .
$$

If we replace the inequality by the equality in Eq. (26) and solve for $\alpha$, we can define adiabatic parameter, which describes how fast the crossing is compared to the fully adiabatic condition:

$$
\alpha_{1} \approx\left(\frac{\epsilon}{4 \pi \Delta_{N L}\left|\Delta_{e}\right|}\right)^{2 / 3},
$$

where $\epsilon$ is the change of $\Delta_{L}$ per revolution and $\kappa \gg 1$ is assumed again. To follow the island center so that it will be trapped, a particle must have sufficiently rapid amplitude change, i.e.,

$$
\alpha \geq \alpha_{1} .
$$

If a Gaussian amplitude distribution is assumed for the trapping efficiency, the particle density is given by

$$
\begin{gathered}
D\left(\alpha^{1 / 2}\right) d \alpha^{1 / 2}=2 \alpha d \alpha^{1 / 2} \exp (-\alpha), \\
\int_{0}^{\infty} D\left(\alpha^{1 / 2}\right) d \alpha^{1 / 2}=1,
\end{gathered}
$$

where the second equation is the normalization in which the total number of particles is unity. With Eqs. (28)-(30), trapping efficiency is

$$
P_{T}=\frac{A}{\pi\left(\alpha_{s}^{1 / 2}\right)^{2}} \exp \left(-\alpha_{1}\right),
$$

where $\alpha_{s}$ is "typical trapping amplitude,"

$$
\alpha_{s}^{1 / 2} \approx \begin{cases}\alpha_{1}^{1 / 2}, & \text { if } \alpha_{1}>1, \\ 1, & \text { if } \alpha_{1}<1 .\end{cases}
$$

The meaning of Eq. (32) is as follows. When $\alpha_{1}>1$, a typical particle being trapped will be around the lower limit of $\alpha_{1}^{1 / 2}$. When $\alpha_{1}<1$, the denser distribution satisfying $\alpha>\alpha_{1}$ is around $\alpha=1$.

In Eq. (31), the factor $A / \pi\left(\alpha_{s}^{1 / 2}\right)^{2}$ represents a normalization by total island area divided by beam radius of "typical trapping amplitude." For third order resonance crossing, Eq. (31) can be expressed as

$$
P_{T}=\frac{\pi}{\sqrt{2}} \kappa^{-(1 / 2)} \alpha_{s}^{-(1 / 4)} \exp \left(-\alpha_{1}\right)
$$


In order to compute trapping efficiency with Eq. (33), the following values are used. The crossing speed is determined from the slope of tune relative to beam energy described in Sec. II A together with the energy gain per turn, the beam emittance is found from the analysis given in Sec. III B, and the driving term and nonlinear detuning have been derived from simulations as will be explained in Sec. IVA 2. These values are summarized in Table IV.

\section{Simulation}

Simulation is based on field calculation and numerical tracking of particle motions. The field calculation was performed with TOSCA and it includes the current errors and the rf cavity cores described in Sec. II C. Tracking is based on numerical integration with the fourth order Runge-Kutta method. We employed a single particle simulation since the space charge effect is negligible in this study.

In order to confirm whether the particle trapping model is applicable to our case or not, numerical tracking with acceleration was carried out. Figure 13 shows an evolution of particle distribution in phase space for no current error and the energy gain of $0.13 \mathrm{keV} / \mathrm{turn}$. It is clear that some particles are trapped by islands and transported to larger amplitudes. We have confirmed that the picture of particle trapping model works in a third order resonance crossing.

The conditions for this calculation are as follows. Initial particle distributions are assumed to be Gaussian and the beam emittance is taken from Table IV. The motion is confined on the median plane and energy gain per turn is constant so that vertical and longitudinal motions are not included. Their effects will be discussed later. The trapping efficiency obtained by simulation has a statistical error. As described in Ref. [8], it is

$$
\delta P_{T}= \pm \frac{\sqrt{m(1-m / n)}}{n}
$$

where $n$ is the number of total particles and $m$ is the number of trapped particles. For $n=350$, the statistical error is expected to be less than or comparable to the error of experimental results.

Since it is not possible to find the strength of the driving term and the amount of nonlinear detuning from beam study, they were estimated from the calculated field. Two approaches are possible: one is to calculate multipole coefficients of the field directly and integrate them throughout the ring, and the other is to locate the fixed points in phase space and relate their positions to the driving term and the nonlinear detuning. The first is straightforward but we employed the second approach for the following reason. It is difficult to estimate multipole coefficients with sufficient accuracy in the fringe field region. Furthermore, the driving term may be affected by the so-called feed-down effect of higher order multipoles, and one may also need to include the second-order effects of sextupoles [14]. Figure 14 shows a phase space with no current error. Even with no current error, COD induced by rf cores excites the third order resonance, and we found three unstable fixed points and three outer islands in addition to the central stable area.

\section{Comparison}

Trapping efficiencies versus energy gain per turn are presented in Fig. 15. Theoretical predictions for three different values of current error are much lower than the observed ones. We have examined the possibility that a small $\kappa$ results in an underestimate of trapping efficiency since $\kappa \gg 1$ is assumed in the model. The range of $\kappa$ in our study is from 1.6 to 2.1. We calculated Eqs. (23) and (27) without any approximation and applied them to Eq. (33). The results are, however, essentially the same to Fig. 15. Therefore, a small $\kappa$ does not explain the discrepancy directly. However, in Ref. [8], they says the normalization in the equation of trapping efficiency fails when $\kappa$ is small. For instance, the factor in Eq. (33) should be

$$
\frac{\pi}{\sqrt{2}} \kappa^{-(1 / 2)} \alpha_{s}^{-(1 / 4)} \lesssim 1 .
$$

That might be one of the reasons for the discrepancy. At the same time, the simulation study ensures that the model works qualitatively even for our small $\kappa$ case, and we found that the observed trapping efficiencies are in good agreement with the results from simulation.

\section{B. Vertical and longitudinal motion}

Although the effects of vertical and longitudinal motions are not included in the model, they may be important since a beam must have a finite vertical as well as longitudinal

TABLE IV. Parameters for theoretical calculation. $E_{g}$ is the energy gain per turn. For beam emittance, the adiabatic damping from 110 to $130 \mathrm{keV}$ is assumed. When Gaussian distribution is assumed, the average beam emittance is one-half of the $87 \%$ beam

\begin{tabular}{|c|c|c|c|}
\hline Current error & $0 \%$ & $-2 \%$ & $-3 \%$ \\
\hline Crossing speed & $-8.3 \times 10^{-4} E_{g}$ & $-7.0 \times 10^{-4} E_{g}$ & $-6.9 \times 10^{-4} E_{g}$ \\
\hline $87 \%$ beam emittance & $760 \pi \mathrm{mm} \mathrm{mrad}$ & $600 \pi \mathrm{mm} \mathrm{mrad}$ & $720 \pi \mathrm{mm} \mathrm{mrad}$ \\
\hline Driving term & $1.30 \times 10^{-2} \mathrm{~m}^{-1 / 2}$ & $1.04 \times 10^{-2} \mathrm{~m}^{-1 / 2}$ & $1.51 \times 10^{-2} \mathrm{~m}^{-1 / 2}$ \\
\hline Nonlinear detuning & $3.63 \times 10^{-1} \mathrm{~m}^{-1}$ & $4.06 \times 10^{-1} \mathrm{~m}^{-1}$ & $4.22 \times 10^{-1} \mathrm{~m}^{-1}$ \\
\hline
\end{tabular}
emittance. 

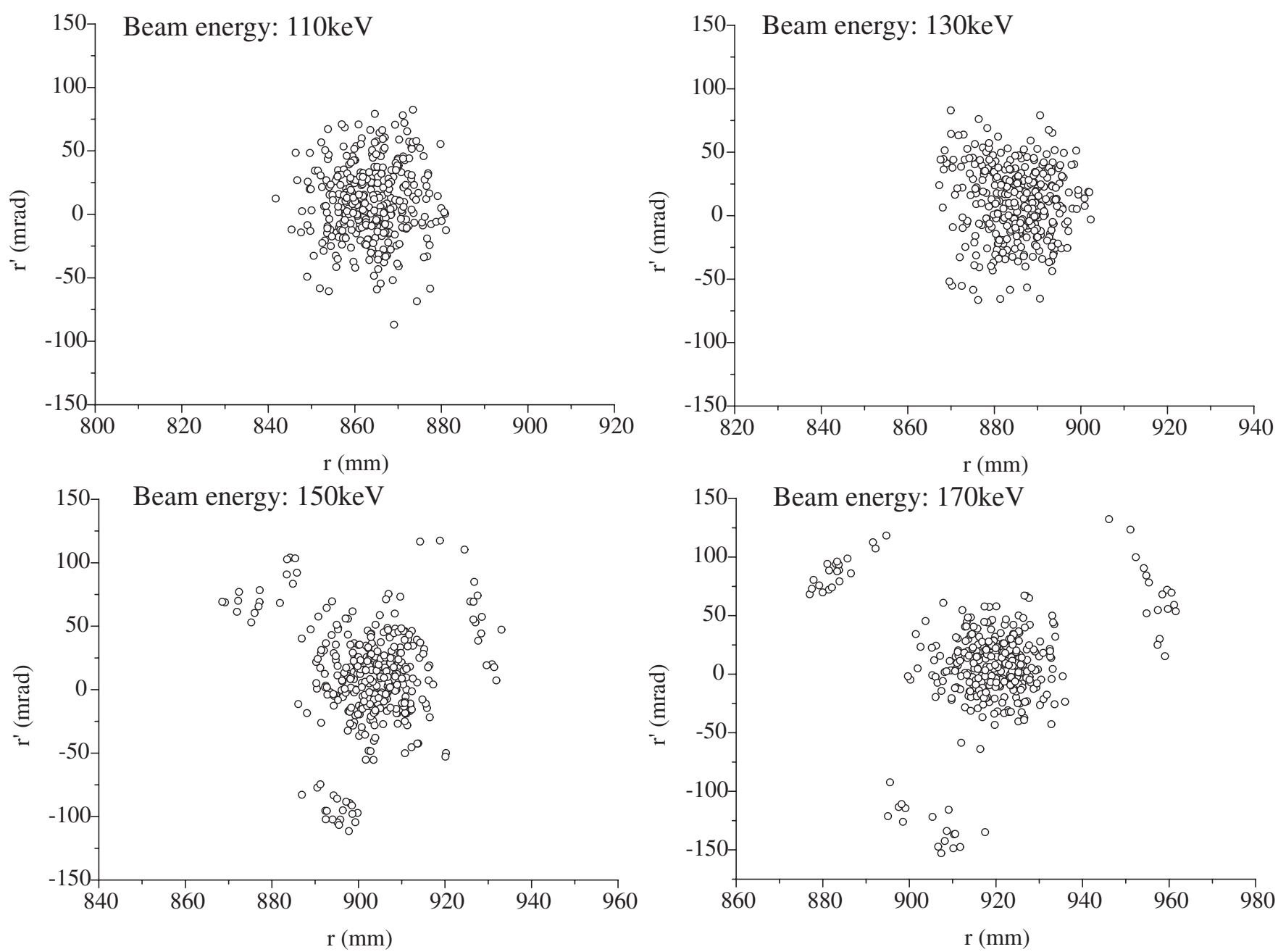

FIG. 13. Simulation results; an evolution of particle distribution during crossing.

emittance. We will discuss the effect of these motions, separately.

\section{Effect of vertical motion}

Vertical motion is a possible cause for modification of the nonlinear detuning. With vertical motion, a contribution from octupole component to the perturbation Hamiltonian is

$$
H_{\mathrm{oct}}^{(1)}=O(\theta)\left(\frac{3}{8} a_{x}^{2} \beta_{x}^{2}-\frac{3}{2} a_{x} a_{y} \beta_{x} \beta_{y}+\frac{3}{8} a_{y}^{2} \beta_{y}^{2}\right),
$$

where $a$ is the canonical variable for oscillation amplitude, $\beta$ is the linear lattice parameter of the ring, and subscripts $x$ and $y$ denote horizontal and vertical directions, respectively. Taking a partial differentiation of Eq. (36) with respect to $a_{x}$, we get

$$
\varphi_{x}^{\prime}=\frac{\partial H_{\mathrm{oct}}^{(1)}}{\partial a_{x}}=O(\theta)\left(\frac{3}{4} a_{x} \beta_{x}^{2}-\frac{3}{2} a_{y} \beta_{x} \beta_{y}\right),
$$

where $\left(a_{x}, \varphi_{x}\right)$ are the canonical variables and $\varphi_{x}^{\prime}$ is the

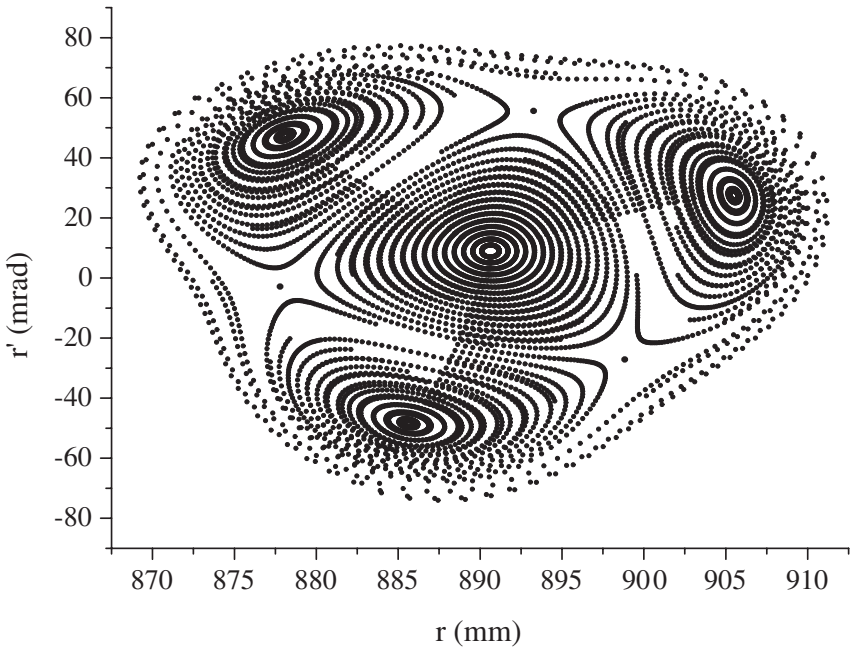

FIG. 14. Fixed points in phase space for the current error of $0 \%$. 


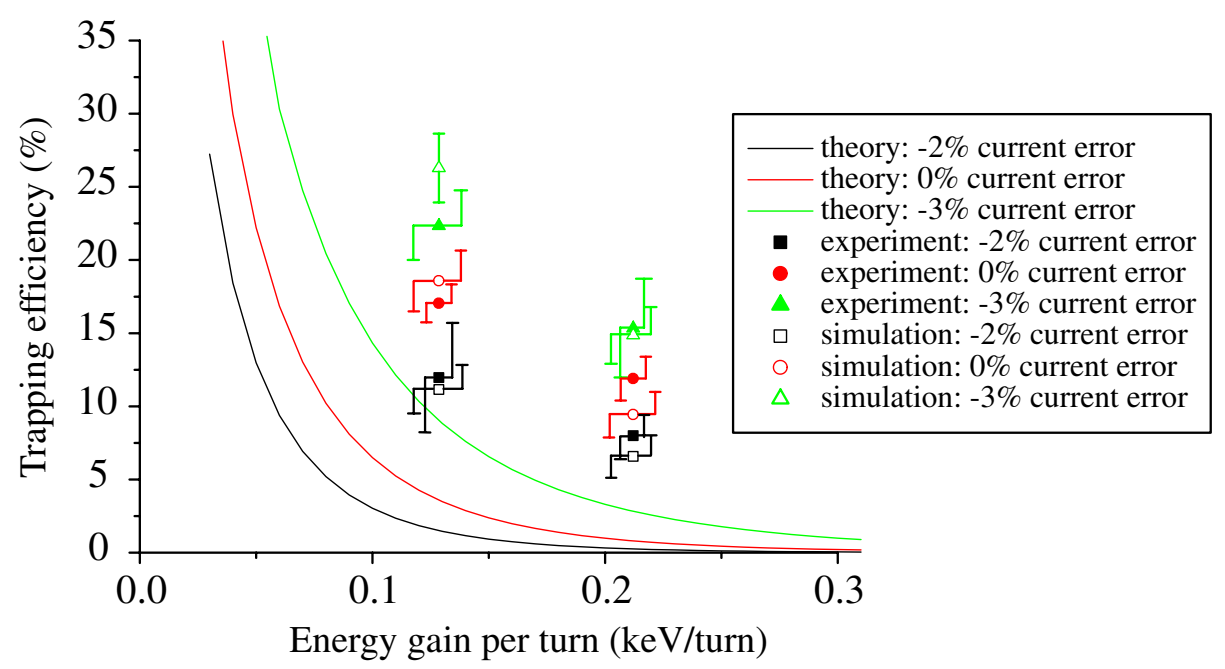

FIG. 15. (Color) Summary of trapping efficiencies.

derivative with respect to $\theta$. This equation shows that the nonlinear detuning is a combination of $a_{x}$ and $a_{y}$ terms. Since $a$ and $\beta$ are always positive, the horizontal detuning (the first term) is weakened by the second term representing the contribution of vertical motion or, if the second term is larger than the first term, the sign of detuning will be reversed.

We evaluated approximately the effect of vertical motion for the case when the maximum trapping efficiency is observed, for instance, the current error of $-3 \%$ and the energy gain per turn is $0.13 \mathrm{keV} /$ turn. We assume that $a_{x}$ and $a_{y}$ are $980 \pi \mathrm{mm} \mathrm{mrad}$ and $30 \pi \mathrm{mm} \mathrm{mrad}$, respectively. They are the emittance corresponding to "typical trapping amplitude" defined by Eq. (32) and the vertical average emittance, respectively, at the crossing energy. The vertical average emittance has been taken from the previous beam study [12]. Figure 16 shows the beta function for one cell. In the region of focusing and defocusing magnets, average beta functions are $\left(\beta_{x}, \beta_{y}\right)=$ $(0.73 \mathrm{~m}, 0.46 \mathrm{~m})$ and $\left(\beta_{x}, \beta_{y}\right)=(0.38 \mathrm{~m}, 0.52 \mathrm{~m})$, respec-

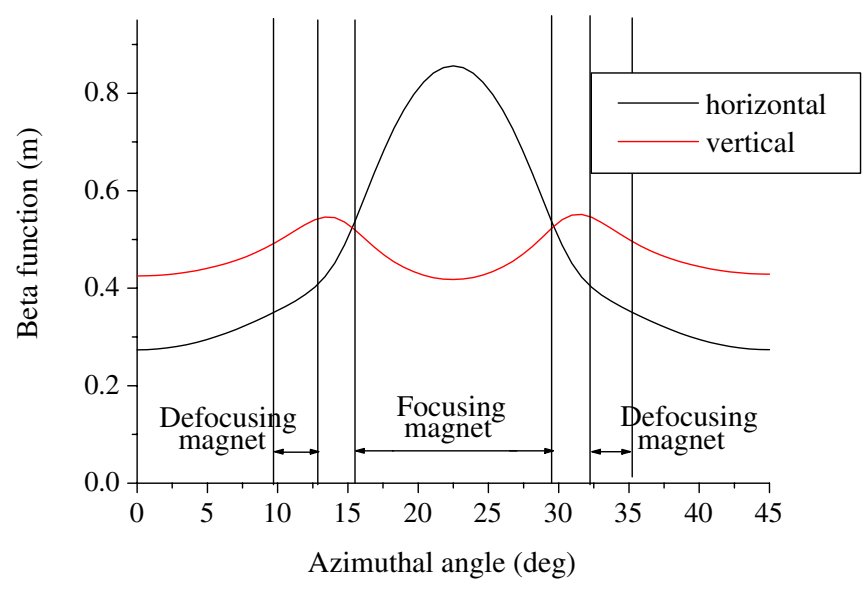

FIG. 16. (Color) Beta function for one cell (without COD). tively. With these values, the terms in Eq. (37) are calculated and they are listed in Table V. In this calculation, we assumed that octupole component is expected to be mostly in the main body of focusing and defocusing magnets.

We see that the contribution of vertical motion is less than $5 \%$ of the horizontal contribution. The effect of vertical motion must be negligible in the present case. Generally speaking, in the radial scaling type of FFAG [2], the contributions of vertical motion in focusing and defocusing magnets tend to cancel each other.

\section{Effect of longitudinal motion}

Crossing speed for particles in a bunched beam is not the same because of synchrotron oscillation. A fluctuation of crossing speed may or may not cause a net change in trapping efficiency. If the period of synchrotron oscillation is much shorter than the time scale for particle trapping, the longitudinal motion should not be significant as the average crossing speed for any particle would nearly be the same as that of synchronous particle. If the period of synchrotron oscillation is much longer, different particles would have different crossing speed depending on the phase at the time of crossing. In the latter case, trapping

TABLE V. Estimation of the effect of vertical motion. Terms in Eq. (37) are calculated for focusing and defocusing magnet, respectively, and normalized by the first term for focusing. Note that the FD ratio, that is, the ratio of focusing field to defocusing field, is now 2.43. The integrated octupole component in the focusing magnet is then 2.43 times larger than the defocusing magnet.

\begin{tabular}{lccc}
\hline \hline & First term & Second term & Second/First \\
\hline Focusing & 1.0 & -0.039 & -0.039 \\
Defocusing & -0.11 & 0.0093 & -0.084 \\
Sum & 0.89 & -0.029 & -0.033 \\
\hline \hline
\end{tabular}


TABLE VI. Estimation of synchrotron phase advance during trapping process.

\begin{tabular}{lc}
\hline \hline \multicolumn{1}{c}{ Parameter } & Value \\
\hline Synchrotron tune & $1.5 \times 10^{-2}$ \\
Number of turns during trapping & 44 \\
Phase advance during trapping & $240 \mathrm{deg}$ \\
\hline \hline
\end{tabular}

efficiency should increase because the dependence of efficiency is not proportional to the acceleration speed and its second derivative with respect to the energy gain is positive as seen in Fig. 15. The latter case is the maximum estimate of the effect of longitudinal motion.

Table VI shows an estimate of how much the phase of synchrotron oscillation advances during the trapping process. The period of synchrotron oscillation is shorter than the time scale for trapping. Therefore the effect of synchrotron oscillation may increase trapping efficiency but it might be less than the maximum estimate. The effect is evaluated with particle tracking simulation more precisely. We again examine the case for which the maximum trapping was observed, the current error of $-3 \%$ and the energy gain per turn of $0.13 \mathrm{keV} /$ turn. In order to clarify the dependence of trapping efficiency on the synchrotron oscillation phase, the initial longitudinal condition is chosen as shown in Fig. 17. Table VII lists results of the tracking simulation.

It is seen that the trapping efficiency varies with the initial phase. However, the efficiency averaged over phase is almost the same as that of the synchronous initial condition. Even when the quadrupole mode of synchrotron oscillation is strong, the averaged efficiency should not vary much because particles in antiphase balance each other. We can neglect the effect of synchrotron oscillation, at least when the bunch length is less than or equal to that of this experiment.

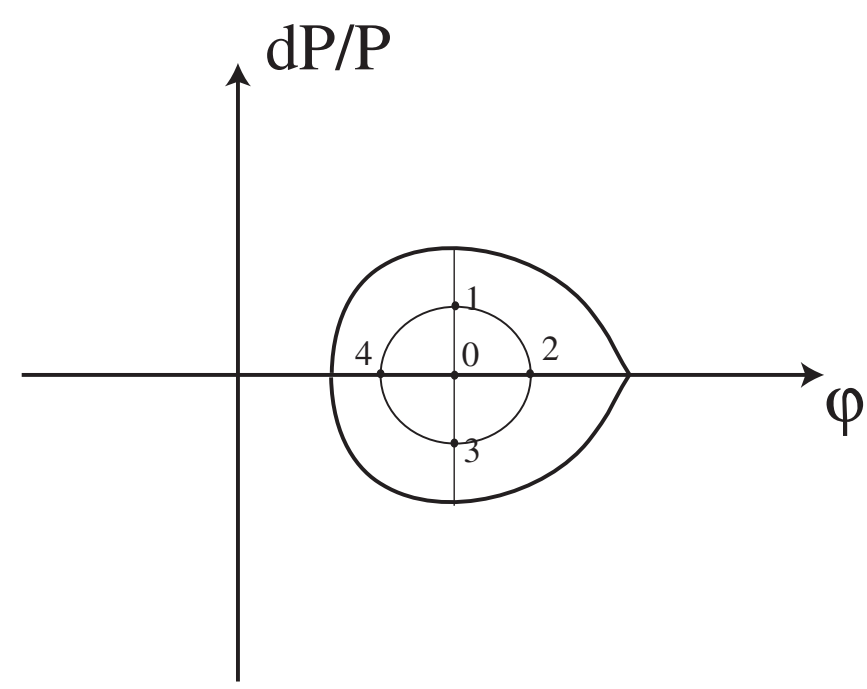

FIG. 17. Initial points of particle in simulation.
TABLE VII. Simulation results for longitudinal motion.

\begin{tabular}{lccc}
\hline \hline Initial condition & Trapped & Total & Efficiency \\
\hline 0 & 92 & 350 & $26.3 \pm 2.4$ \\
1 & 90 & 350 & $25.7 \pm 2.3$ \\
2 & 87 & 350 & $24.9 \pm 2.3$ \\
3 & 96 & 350 & $27.4 \pm 2.4$ \\
4 & 107 & 350 & $30.6 \pm 2.5$ \\
Average for 1-4 & 95 & 350 & $27.1 \pm 1.2$ \\
\hline \hline
\end{tabular}

\section{Criterion to avoid trapping}

The most important purpose of our study is to find the criterion to avoid any deterioration in beam quality arising from resonance crossing. We observed in our study that there is no clear evidence of adverse effects on the beam when the crossing speed is sufficiently large. However, the crossing speed is not the only factor affecting the trapping efficiency. The adiabatic parameter, $\alpha_{1}$ in Eq. (27), is a measure of how fast the center of islands move compared with the speed of the particle moving in phase space away from center. It contains all the relevant factors, that is, crossing speed, strength of driving term, amount of nonlinear detuning, and beam emittance. We employ the adiabatic parameter as an index for avoiding beam deterioration due to resonance crossing. Figure 18 shows the observed trapping efficiency as a function of the adiabatic parameter.

We found from Fig. 18 that particles are not trapped when the adiabatic parameter is more than 7. At the same time, $\kappa$ in Eq. (33) is another factor to determine trapping efficiency. A few percent current error is unrealistically large from the viewpoint of magnetic field for accelerator, and the large driving term is excited. Even with no current error, the driving term is comparable to the ones with a few percent current error because the field error due to the $\mathrm{rf}$

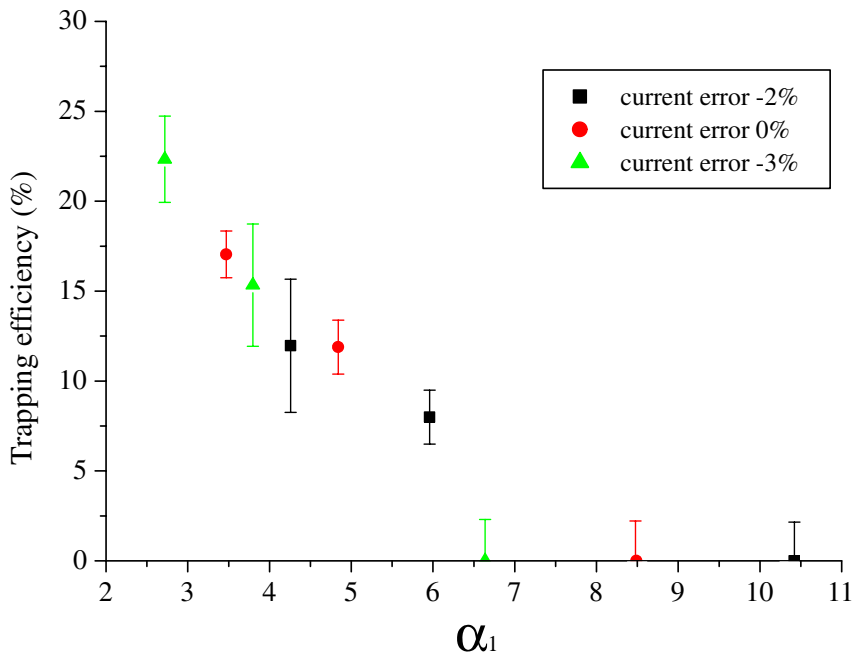

FIG. 18. (Color) Trapping efficiency as a function of adiabatic parameter. 
cores is relatively large for the low energy beam. This fact results in small $\kappa$. In general, smaller values of adiabatic parameters will be permitted when $\kappa$ is larger than the ones in this study. A crossing should then be harmless when the adiabatic parameter is more than 7 .

\section{CONCLUSION}

Beam dynamics of resonance crossing has been studied with the experiment at PoP FFAG. In this paper, the details of experiment are described. The experiment shows that no substantial effect on the beam results from resonance crossing when the crossing is relatively fast but part of the beam is transported to a larger amplitude when the crossing is relatively slow. It is clearly seen with the simulation study that part of the beam is trapped by islands in phase space when crossing is relatively slow. Then, the theoretical model of particle trapping should be applicable to our case and its prediction agrees with the experimental results qualitatively. The quantitative discrepancy could be explained by the fact that the normalization in the equation of trapping efficiency fails with the parameters of this experiment. At the same time, there is a good agreement between the prediction from simulation and the experimental results. We have derived the adiabatic parameter to be used as a criterion for avoiding a deterioration in beam quality when a resonance is crossed. This parameter contains all the relevant factors so that one can judge whether a crossing should be regarded as "slow" or "fast" for given conditions. We conclude that a crossing should be harmless when the adiabatic parameter is more than 7 .

\section{ACKNOWLEDGMENTS}

We are deeply grateful to Akira Takagi, Dr. Masahiro Yoshimoto, and Yujirou Yonemura for their assistance on the experiment. We also thank Dr. Francois Meot for his help during his stay in KEK.

\section{APPENDIX: CORRECTION OF BEAM LOSS}

In the energy range of PoP FFAG, 50 to $500 \mathrm{keV}$, beam loss by the residual gas is not negligible, especially near injection energy. The background vacuum pressure in PoP FFAG is about $1 \times 10^{-7}$ Torr while the pressure during operation is about $6 \times 10^{-7}$ Torr. Hydrogen from the iron source is the main component of the residual gas during operation. In this energy range, the dominant interaction between proton beam and hydrogen gas is a charge transfer since the cross section of an elastic scattering is negligible compared with that of charge transfer. The cross section of charge transfer is shown in Fig. 19.

With only single interaction of charge transfer, a particle loses its charge and will be lost. Therefore, the beam emittance does not grow but the beam intensity decreases in this process. For correction, it is sufficient to normalize intensity by the beam loss rate. Specifically, the procedure

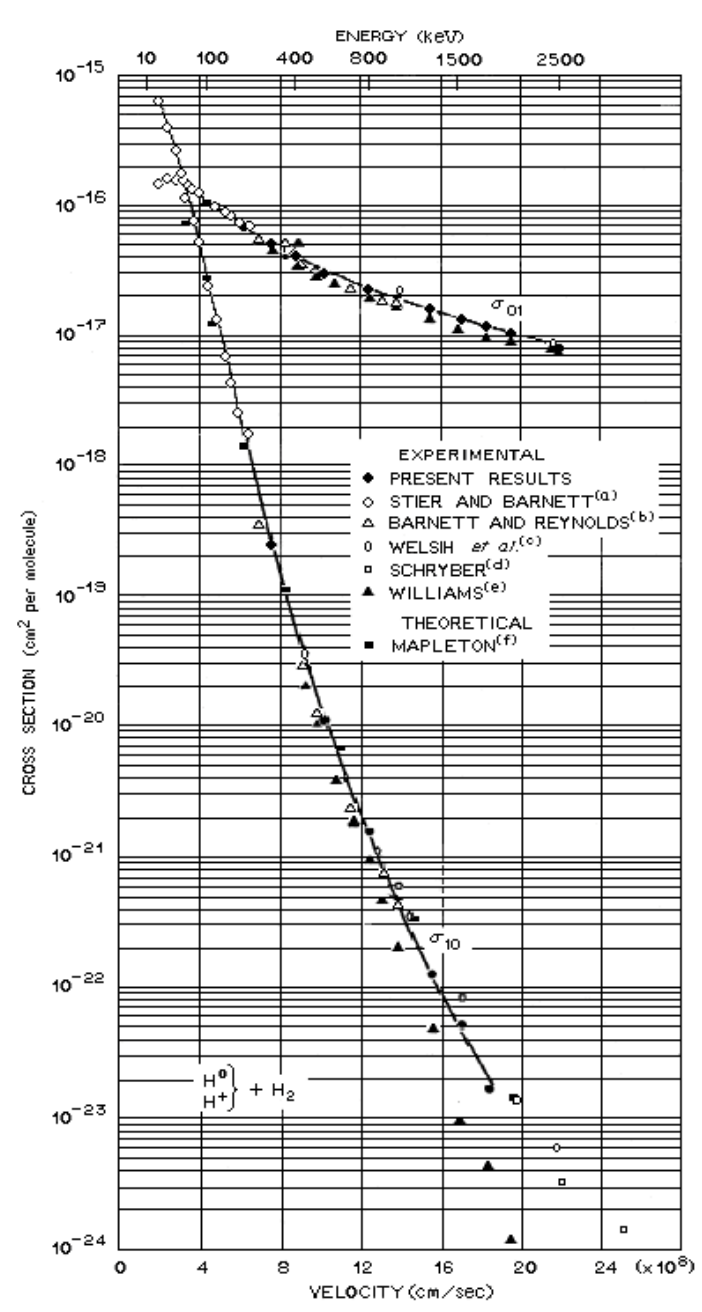

FIG. 19. Cross section of charge transfer (taken from Ref. [15])

is to find a suitable fitting function and apply it to the beam intensity curve.

When the beam energy is unchanged, the intensity curve should simply follow an exponential decay. However, the beam is being accelerated and a suitable fitting function is not simply exponential anymore. A lifetime of exponential decay depends on beam energy. Therefore the lifetime changes during acceleration and it should be derived as a function of beam energy. Since the beam intensity was obtained as a function of turn number, a function of turn number is more convenient. The lifetime is inversely proportional to the cross section,

$$
\tau \propto \frac{1}{S(n)},
$$

where $S(n)$ is the cross section as a function of turn number $n$ during acceleration. As shown in Fig. 19, in the energy range of PoP FFAG, the cross section is almost proportional to the particle velocity in logarithmic scale, i.e.,

$$
\ln S=A_{1} v,
$$


where $S$ is the cross section, $v$ is the particle velocity, and $A_{1}$ is a constant. With nonrelativistic approximation, the relation between the particle velocity and energy can be expressed by

$$
E_{k} \approx \frac{1}{2} m v^{2}
$$

where $E_{k}$ is the kinetic energy and $m$ is the rest mass of proton. Beam energy is given by

$$
E_{k}=E_{\text {inj }}+n E_{g},
$$

where $E_{\text {inj }}$ is the injection beam energy, $n$ is the number of turns during acceleration, and $E_{g}$ is the energy gain per turn. From Eqs. (A2)-(A4), we can obtain

$$
S=\exp \left(A_{2} \sqrt{E_{\mathrm{inj}}+n E_{g}}\right)
$$

where $A_{2}$ is a constant. The lifetime is then

$$
\tau=\frac{A_{3}}{\exp \left(A_{2} \sqrt{E_{\text {inj }}+n E_{g}}\right)}
$$

where $A_{3}$ is also a constant. The beam intensity can be expressed as

$$
I=I_{0} \exp \left(-\frac{n}{\tau}\right)
$$

where $I_{0}$ is the initial intensity. Finally, the fitting function can then be expressed in the form with four fitting parameters $(P 1, P 2, P 3, P 4)$,

$$
I=P_{1} \exp \left[-P_{2} n \exp \left(P_{3} \sqrt{E_{\text {inj }}+n P_{4}}\right)\right]
$$

[1] T. Ohkawa, originally proposed at the Symposium on Nuclear Physics of the Physical Society of Japan in 1953 (private communication).

[2] K. R. Symon, Phys. Rev. 103, 1837 (1957).

[3] D. Trbojevic, E. D. Courant, and M. Blaskiewicz, Phys. Rev. ST Accel. Beams 8, 050101 (2005).

[4] P. A. Sturrock, Ann. Phys. (Berlin) 3, 113 (1958).

[5] W. P. Lysenko, Part. Accel. 5, 1 (1973).

[6] G. Guignard, CERN-76-06, 1976.

[7] R. Baartman, in Proceedings of the 10th International Conference on Cyclotrons and Their Applications, East Lansing, 1984 (IEEE, New York, 1984), p. 40.

[8] A. W. Chao and M. Month, Nucl. Instrum. Methods 121, 129 (1974).

[9] M. Giovannozzi et al., in Proceedings of the Nineth European Particle Accelerator Conference, Lucerne, 2004 (EPS-AG, Lucerne, 2004), p. 173.

[10] M. Aiba, Ph.D. thesis, University of Tokyo, 2005.

[11] Y. Sato et al., in Proceedings of the Seventh European Particle Accelerator Conference, Vienne, 2000 (EPS, Vienna, 2000), p. 581.

[12] M. Yoshimoto, Ph.D. thesis, Doshisya University, 2003 (in Japanese).

[13] Y. Mori et al., in Proceedings of the Sixth European Particle Accelerator Conference, Stockholm, 1998 (Institute of Physics, Philadelphia, 1998), p. 299.

[14] R. D. Ruth, in SLAC Summer School, 1985, AIP Conf. Proc. No. 153 (AIP, New York, 1987), p. 150.

[15] L. H. Toburen, M. Y. Nakai, and R. A. Langley, Phys. Rev. 171, 114 (1968). 